\title{
LA FORMACIÓN POR COMPETENCIAS Y LOS ESTUDIANTES: CONFLUENCIAS YDIVERGENCIAS EN LA CONSTRUCCIÓN DEL DOCENTE IDEAL ${ }^{1}$
}

\author{
Rodrigo Asún Inostroza ${ }^{2}$, Claudia Zúñiga Rivas ${ }^{3}$, \\ María Constanza Ayala Reyes ${ }^{4}$
}

\begin{abstract}
RESUMEN
El objetivo de esta investigación fue determinar el nivel de coincidencia existente entre las demandas pedagógicas que el Modelo de Formación por Competencias (MFC) hace a los docentes universitarios y las que expresan los estudiantes. Se ha construido un modelo de lo que constituiría un «Docente Universitario Competente» según el MFC, para compararlo con los discursos de los estudiantes, recogidos en ocho grupos focales en los que participaron 54 estudiantes de la Universidad de Chile. Los resultados indican que si bien hay numerosas áreas de coincidencia entre ambas demandas, existen también importantes divergencias en cuanto a la planificación, evaluación, resultados esperados, responsabilidad y didáctica del proceso educativo, encontrándose que los estudiantes se muestran más tradicionales en sus demandas que el MFC.
\end{abstract}

Palabras clave: Modelo de Formación por Competencias, docente competente, demandas a la docencia, estudiantes universitarios.

\section{STUDENTS ANDCOMPETENCIESFORMATION: CONVERGENCES AND DIVERGENCES IN CONSTRUCTING THE IDEAL TEACHER}

\begin{abstract}
This research was aimed to determine the level of matching between the pedagogical demands the Competencies Formation Model (CFM) puts on university teachers and those expressed by university students. For this, we have constructed a model of what would constitute a "Competent University Teacher" according to CFM and compared it to discourses of students participating in eight focus groups including 54 students from University of Chile. Results indicate that while there are numerous overlapping areas between both demands, there are also relevant differences in understanding planning, evaluation, outcomes, responsibility and teaching of the educational process, showing that students are more conservative in their demands than the model.
\end{abstract}

Keywords: Competencies Formation Model, competent teacher, demands on teaching, university students

1 Este artículo reporta los resultados de un proyecto de investigación financiado por el Programa Pulso de la Facultad de Ciencias Sociales de la Universidad de Chile y por el proyecto «Construcción y validación de un banco de ítems para la evaluación de la calidad de la docencia por parte de estudiantes universitarios» (Soc 08/06-2), de la Vicerrectoría de Investigación y Desarrollo de la Universidad de Chile.

2 Departamento de Sociología, Facultad de Ciencias Sociales, Universidad de Chile, Santiago, Chile. Dirección Postal: Ignacio Carrera Pinto 1045, Ñuñoa, Santiago, Chile. E-mail: rasun@ uchile.cl.

3 Departamento de Psicología, Universidad de Chile, Santiago, Chile.

4 Departamento de Sociología, Universidad de Chile, Santiago, Chile. 


\section{LA FORMACIÓN POR COMPETENCIAS Y LOS ESTUDIANTES: CONFLUENCIAS Y DIVERGENCIAS EN LA CONSTRUCCIÓN DEL DOCENTE IDEAL}

\section{Introducción}

El Modelo de Formación por Competencias (MFC) es presentado por sus partidarios como un nuevo paradigma educativo que puede hacer más efectivo el proceso de enseñanza-aprendizaje en el contexto actual (De Miguel, 2005). No obstante, esa imagen no es aceptada unánimemente por la comunidad de expertos en educación, y es posible encontrar autores que incluso discuten tanto la novedad de este enfoque -Díaz-Barriga (2006), por ejemplo, plantea que esta orientación es solo la última de una serie de propuestas pedagógicas que propugnan innovaciones similares-como el que se ponga tanto acento en la satisfacción de las demandas del mercado laboral y/o en la transmisión de procedimientos técnicos (Gómez y Alzate, 2010).

Independientemente de que esas críticas hayan sido o no adecuadamente respondidas (véase, por ejemplo, cómo Van Vucht Tijssen y De Weert (2005) niegan que adoptar este modelo implique necesariamente optar por la simple racionalidad instrumental), no es posible dudar del impulso que este enfoque ha ido alcanzando en la educación superior en los últimos años y la legitimidad que ha adquirido como perspectiva pedagógica. Así por ejemplo, la construcción del Espacio Europeo de Educación Superior y el acuerdo de Bolonia optan por esta perspectiva docente (Fernández-Salinero, 2006) y muchas universidades, a impulsos propios o externos, han adaptado o se encuentran adaptando sus procesos formativos a las recomendaciones y lineamientos sugeridos por este modelo.

Es posible afirmar que la implementación del MFC implica hacer modificaciones en dos niveles del sistema formativo: a) en primer lugar, requiere un rediseño de la estructura curricular, de manera de pasar de un enfoque basado en el logro de objetivos, a la 
formación de competencias, lo que implica repensar completamente los contenidos a incluir y su interrelación (Tardif, 2003; b) exige una nueva forma de trabajo al docente de aula, que debe reorientar su quehacer de manera de transitar desde un enfoque pedagógico centrado en el profesor a otro centrado en el estudiante (Tejada, 2002).

En relación con la segunda serie de transformaciones existe un importante volumen de evidencia que señala que una docencia centrada en el estudiante tiene consecuencias positivas tanto en la magnitud como en la profundidad de los aprendizajes de los alumnos (González, 2010). No obstante, una variable que podría asumir un rol mediador clave en la relación entre esta perspectiva docente y sus efectos positivos puede ser el grado en el que este nuevo estilo de docencia es coherente con las expectativas que tienen los estudiantes respecto de la conducta de los profesores, ya que la no convergencia entre ambas puede conspirar contra su eficacia y disminuir las probabilidades de éxito en la implementación del MFC o de cualquier otra propuesta de innovación docente.

Si bien existen algunos precedentes en el intento de determinar las demandas que los estudiantes universitarios hacen a sus profesores, la mayor parte de esos estudios se remite a un relato descriptivo, sin compararlo con algún modelo pedagógico (Casero, 2010; Gallardo y Reyes 2010; Giné, 2008; Greimel-Fuhrmann y Geyer, 2003; Jahangiri y Mucciolo, 2008) o utilizan metodologías cuantitativas que restringen las posibilidades de respuesta de los estudiantes y, por lo tanto, no permiten realizar una comparación exhaustiva con algún modelo educativo (Chonko, Janner y Davies; Francis, 2006; Okpala y Ellis, 2005; Pozo, Rebolloso y Fernández, 2000).

Excepciones a lo señalado son los estudios de Bedek y Urbanc (2009) y de Gargallo, Sánchez, Ros y Ferreras (2010), quienes plantean la existencia de coincidencias entre las demandas de alumnos universitarios y lo que solicita a los profesores el proceso de Bolonia, o lo que denominan el «modelo de facilitación del conocimiento» (cuya descripción es equivalente a los principios del MFC). El problema de estas investigaciones es que, más allá de lo concluido por los autores, los instrumentos de producción de información empleados 
(basados en preguntas cerradas) no permiten dilucidar en forma clara y detallada el grado de coherencia entre ambas orientaciones.

Por todo lo anterior, es posible afirmar que actualmente la evidencia disponible no es concluyente respecto de la confluencia entre lo que el MFC requiere de los docentes y las demandas de los estudiantes universitarios.

\section{Formulación del problema e hipótesis}

En la presente investigación se construirá la caracterización que hace el MFC de un profesor ideal (o «docente competente», según su propia nomenclatura), para luego contrastar esta descripción con las demandas que formulan los estudiantes universitarios a sus educadores, a fin de constatar las similitudes y divergencias entre ambas orientaciones normativas.

Dados los resultados de estudios previos (Bedek y Urbanc, 2009; Gargallo, Sánchez, Ros y Ferreras, 2010) se hipotetiza que la definición de los atributos de un profesor perfecto es la misma, tanto si ella se construye desde el MFC, como si se le describe desde las demandas de los estudiantes. Ya que se considera discutible la existencia de esta convergencia, el objetivo de esta investigación será poner a prueba esta hipótesis utilizando como evidencia las descripciones que hacen del profesor ideal el MFC y los discursos de los estudiantes de una universidad chilena.

La relevancia de realizar esta comparación radica en que los importantes esfuerzos y recursos que se están destinando actualmente a la introducción de la formación por competencias en la educación superior universitaria exigen realizar estudios socioeducativos respecto de las posibilidades de éxito del proceso y, como ya ha sido señalado por los mismos impulsores de este cambio, la transformación exitosa del sistema educativo solo será posible con la participación y disposición favorable a la innovación de los distintos colectivos que forman parte de las estructuras a modificar (De Miguel, 2006). Por ello, es posible sostener que si no hay un cierto nivel de compatibilidad entre las visiones que los estudiantes tienen de «profesor ideal»y 
el MFC, es muy posible que este último enfrente resistencias que arriesguen el éxito de su implementación.

Si bien la posibilidad de generalizar los resultados de esta investigación está limitada por haberse realizado en una sola universidad, el que esta sea la mayor institución de educación superior chilena y que por su calidad y carácter público acoja a jóvenes provenientes de una importante diversidad social, territorial y cultural permitirá establecer una aproximación a la coherencia entre las demandas de los estudiantes y las del MFC, al menos para el caso chileno.

Con el fin de juzgar si este discurso estudiantil es generalizable a otros países y contextos, en las conclusiones se discutirá su semejanza con lo reportado por otros estudios similares.

A continuación se describe lo que constituye un profesor ideal desde la teoría de formación por competencias, modelo que ha sido construido a partir del análisis de textos elaborados por autores que respaldan esa propuesta pedagógica.

\section{El profesor ideal según la formación por competencias}

En términos generales y siguiendo a De Miguel (2006), podemos señalar que el MFC plantea que la pedagogía debería centrarse no solo en transmitir conocimientos, sino también en entregar destrezas acerca de cómo operar y de cómo disponerse personalmente para la ejecución eficiente de las tareas. En función de ello se adoptará como definición general de competencias la noción de que estas «constituyen estructuras complejas de procesos que las personas ponen en acción-actuacióncreación para resolver problemas y realizar actividades (de la vida cotidiana y del contexto laboral profesional), orientadas a la construcción y transformación de la realidad» (Fernández-Salinero, 2006: 138-139).

Estas estructuras se apoyan en la movilización y utilización eficaces de una variedad de recursos (internos y externos, actitudinales, valóricos y cognitivos), lo que implica que ser competente requiere combinar una serie de destrezas relacionadas con el ser, el hacer y el conocer (Villa y 
Villa, 2007). Respecto del ser, se señala que un profesional o docente competente debe actuar con adecuación a los códigos de conducta, los valores y la ética que regulan su profesión, debe ser responsable con sus obligaciones, tener una correcta imagen de sus capacidades y limitaciones y debe preocuparse por la calidad de su trabajo. En relación con el hacer, se menciona la necesidad de disponer de habilidades generales (dentro de las que se encuentran la capacidad de planificar y diseñar una tarea, integrar y liderar grupos, pensar creativa y críticamente, actuar en forma autónoma y aprender de la práctica, entre otras) y específicas (respecto de las cuales se mencionan el dominar las herramientas tecnológicas de su disciplina, tener la experiencia práctica para operar en ella y manejar técnicas que permitan reconocer y resolver los problemas que enfrenta). Finalmente, acerca del conocer se explicita que para ser competente se debe tener conocimiento significativo y actualizado, tanto de lo que la disciplina juzga en ese momento como verdadero, así como de los debates en curso.

En función de esa caracterización global, podemos señalar que el modelo de formación por competencias formula nueve propuestas específicas de transformación del trabajo de un docente (esta síntesis ha sido construida siguiendo a Bozu y Canto, 2009; De Miguel 2005; De Miguel 2006; Tejada, 2009; Valcarcel, 2005; Zabalza, 2003):

a) En el campo de la planificación del proceso educativo se sugiere pasar de una programación rígida y a priori, que solo considera los contenidos por transmitir y los objetivos de aprendizaje por lograr, a una planificación flexible que se adapte a las condiciones de entrada, motivaciones y aspiraciones del grupo concreto con el que se esté trabajando.

b) En el ámbito del dominio disciplinario se transita desde demandar principalmente que el profesor sea un sabio con un dominio exhaustivo del campo, a solicitar que el académico actúe como un consultor experto capaz de seleccionar, enfatizar, ordenar y significar los contenidos relevantes, en función de un contexto aplicado y relevante para los estudiantes.

c) En términos didácticos se debiera evolucionar desde exigir solamente un expositor claro, capaz de producir una clase magistral informativa, a un conocedor de la didáctica, capaz de 
emplear múltiples técnicas y usarlas en función de sus objetivos y del grupo al que se enfrente.

d) En relación con la actitud del profesor se discurre desde valorar a un docente serio, es decir, fuertemente comprometido con las materias disciplinarias que trabaja, pero que controla la expresión de sus emociones, a un docente centrado en el motivar a sus estudiantes, lo que supone un maestro apasionado, capaz de proyectar en los educandos su entusiasmo por su disciplina y la docencia.

e) Por su parte, en la interacción con los estudiantes se camina desde el modelo de un profesor respetuoso de sus alumnos, pero distante y que toma por sí solo todas las decisiones relacionadas con la asignatura, en función de su saber, a un docente igualmente respetuoso, pero más cercano y capaz de escuchar y permitir opinar e incidir a los estudiantes.

f) Respecto de la evaluación de los aprendizajes se sugiere un cambio desde medir en forma precisa los conocimientos logrados por los estudiantes a considerar, además, el nivel de dominio de las competencias alcanzadas y utilizar las evaluaciones de manera formativa. Esto implica aumentar la gama de instrumentos de evaluación empleados para que estos vayan más allá del examen escrito.

g) Desde el punto de vista de los resultados esperados del proceso formativo se pasa de poner el foco en que los estudiantes manejen contenidos disciplinarios, a lograr que estos desarrollen múltiples y diversas competencias generales y específicas, profundizando en la capacidad de acción profesional y en la transmisión de nuevas estructuras de interpretación.

h) En términos del foco del proceso de enseñanza/aprendizaje se propone un desplazamiento desde un proceso centrado en el profesor y su capacidad de enseñar, a otro orientado hacia el estudiante y el fomento de sus habilidades de aprendizaje autónomo.

i) Finalmente, en relación con la complejidad de las exigencias pedagógicas que se hace al docente se pasa de las demandas simples, centradas en que el profesor actúe responsablemente al planificar la asignatura, dicte clases magistrales informativas y construya exámenes válidos, a requerimientos más complejos 
que se traducen en el ser capaz de conducir un proceso educativo flexible, diverso, interactivo, formativo y centrado en el estudiante.

En suma, para el modelo de formación por competencias un profesor ideal es aquel que asume como su responsabilidad la facilitación de los aprendizajes de los estudiantes y posee los conocimientos disciplinarios y las competencias pedagógicas para planificar y ejecutar una serie flexible de actividades didácticas diversas, adaptadas y centradas en sus estudiantes, con los cuales mantiene interacciones motivadoras, abiertas y cercanas, conducentes al desarrollo de capacidades de autoaprendizaje y habilidades específicas y generales, las que son evaluadas formativamente utilizando diversos dispositivos que le permiten certificar el dominio de competencias que obtienen sus educandos.

La Tabla 1 sintetiza en términos esquemáticos la forma en que se concibe a un profesor ideal desde esta propuesta docente en comparación con la pedagogía tradicional.

Tabla 1: Modelos de profesor ideal

\begin{tabular}{|l|l|l|}
\hline Ámbito pedagógico & $\begin{array}{l}\text { Profesor ideal para la pedagogía } \\
\text { tradicional }\end{array}$ & $\begin{array}{l}\text { Profesor ideal para la formación por } \\
\text { competencias }\end{array}$ \\
\hline Planificación & $\begin{array}{l}\text { Rígida y orientada por contenidos y } \\
\text { objetivos }\end{array}$ & $\begin{array}{l}\text { Flexible y orientada hacia las } \\
\text { condiciones de los alumnos }\end{array}$ \\
\hline Dominio disciplinario & Sabio, con conocimientos exhaustivos & $\begin{array}{l}\text { Consultor experto capaz de seleccionar } \\
\text { y significar }\end{array}$ \\
\hline Didáctica & $\begin{array}{l}\text { Centrada en clases magistrales } \\
\text { informativas }\end{array}$ & $\begin{array}{l}\text { Centrada en múltiples técnicas } \\
\text { adaptadas a los estudiantes }\end{array}$ \\
\hline Actitud & $\begin{array}{l}\text { Seriedad y fuerte implicación con el } \\
\text { tema }\end{array}$ & $\begin{array}{l}\text { Pasión proyectada por el tema y la } \\
\text { docencia }\end{array}$ \\
\hline $\begin{array}{l}\text { Interacción con } \\
\text { estudiantes }\end{array}$ & Basada en el respeto, pero distante & $\begin{array}{l}\text { Basada en el respeto, pero cercana, } \\
\text { capaz de dialogar }\end{array}$ \\
\hline Evaluación & $\begin{array}{l}\text { Centrada en la precisión de mediciones } \\
\text { finales }\end{array}$ & $\begin{array}{l}\text { Centrada en el proceso y de carácter } \\
\text { formativo }\end{array}$ \\
\hline Resultados esperados & Manejo de contenidos disciplinarios & $\begin{array}{l}\text { Desarrollo de competencias generales } \\
\text { y específicas }\end{array}$ \\
\hline Foco del proceso & Capacidad del docente de enseñar & $\begin{array}{l}\text { Capacidad del estudiante de aprender, } \\
\text { cada vez más autónomamente }\end{array}$ \\
\hline $\begin{array}{l}\text { Complejidad de las } \\
\text { exigencias pedagógicas }\end{array}$ & Exigencias pedagógicas simples & Exigencias pedagógicas complejas \\
\hline
\end{tabular}

Fuente: Elaboración propia 


\section{Método}

Para determinar las demandas que los estudiantes formulan a sus docentes se optó por realizar una investigación cualitativa utilizando el grupo focal como técnica de producción de datos, pues dicha estrategia permite acceder en forma abierta y profunda a los contenidos y valoraciones que los sujetos despliegan respecto de los temas investigados, permitiendo los intercambios grupales y discusiones acerca de sentimientos, actitudes, creencias y experiencias (Morgan, 1990).

La producción de datos se realizó durante el año 2010, utilizando un muestreo estructural para seleccionar alumnos de 16 carreras de la Universidad de Chile, agrupadas en cuatro categorías: humanidades, artes, ciencias y salud, para así obtener la opinión de la máxima diversidad de participantes. En cada categoría se realizaron dos grupos, uno con alumnos de primer y segundo año y otro con estudiantes de tercer y cuarto año.

Así, se realizaron ocho grupos focales en total, todos con alumnos de ambos géneros, en los que participó un total de 54 estudiantes.

La selección de los participantes fue no probabilística e intencional, ya que la muestra respondió a un criterio de comprensión y pertinencia y no a uno de representatividad estadística (Dávila, 1993).

La pauta para la discusión en los grupos focales fue diseñada, puesta a prueba, evaluada y modificada en función de los resultados de una investigación piloto, que contempló la realización de cuatro grupos focales en una sola facultad de la misma universidad (Facultad de Ciencias Sociales).

Los grupos fueron grabados, transcritos y analizados según los procedimientos de análisis de contenido (Taylor y Bogdan, 1992), lo que implicó clasificar las afirmaciones de los estudiantes en redes de categorías jerárquicas que permitieron organizar y determinar las demandas que realizan a sus docentes. Las categorías fueron 
construidas durante el estudio piloto ya descrito, pero adaptadas a los resultados de la presente investigación. La categorización de las frases fue realizada por los investigadores utilizando el software Atlas. ti 5.0 (2004).

\section{Resultados}

\subsection{Planificación del proceso educativo (rigidez vs. flexibilidad)}

En el discurso estudiantil son pocas las referencias a este tema, por lo que parece poco relevante para la definición de un buen profesor desde la perspectiva de los alumnos. Esto posiblemente no indique que el tema sea esencialmente poco importante, sino probablemente tiene relación con que gran parte del proceso de planificación docente queda fuera de la mirada de los estudiantes, quienes solo pueden inferirlo a partir del desempeño del profesor en la sala de clases.

No obstante, cuando este tema se menciona, tienden a aparecer algunas peticiones relacionadas con realizar una docencia más «contextualizada». Así, los estudiantes solicitan que sus profesores ejemplifiquen los contenidos con temas aplicados, contingentes o cercanos al mundo laboral.

Otra de las demandas que aparece en este ámbito, es que los profesores adapten su discurso al nivel de conocimientos previos del grupo curso:

El profe trataba de aplicar las cosas y además tenía la noción de que no todos estábamos al mismo nivel, siempre hay gente que en física y matemática, ya saben todo y van a la clase a ampliar su conocimiento general, pero hay otros, por ejemplo, a mí me costaba más y el profesor no tenía ningún problema después de clase en quedarse y responder las dudas (estudiante Facultad de Ciencias Físicas y Matemáticas, tercer año).

Sin embargo, la demanda más recurrente en este tema se concentra en solicitar, como un "mínimo indispensable», que el profesor sea «ordenado», es decir, que exista una secuencia de 
contenidos programada a priori y coherente (sin saltos, divagaciones o reiteraciones no justificadas) que el estudiante pueda comprender y seguir:

[Es importante] el tema como de la metodología, o sea, como un orden, porque de repente los profes saltan de un momento a otro y ¿qué pasó al medio? o tenían un programa y de repente hablan cuestiones que tú no entiendes porque en el fondo no notaste qué pasó (estudiante Facultad de Filosofía y Humanidades, cuarto año).

En suma, la demanda estudiantil parece ser una planificación con flexibilidad limitada, donde se pueden hacer modificaciones a la planificación original, pero solo si se respeta en lo esencial dicha planificación. Esto parece indicar una coincidencia solo parcial con lo propugnado por el MFC.

\subsection{Dominio de contenidos (sabio exhaustivo vs. consultor experto)}

En este ámbito los estudiantes exigen unánimemente y también con carácter de «mínimo indispensable», que el docente posea un conocimiento actualizado y profundo de los contenidos del curso.

Yo no dudo de que el profesor que me hace una clase en la Universidad de Chile no tenga el conocimiento que requiere, ni tampoco dudo que sea el conocimiento más actualizado al respecto (estudiante Facultad de Medicina, primer año).

Por ello, cuando hablan del nivel de dominio disciplinario de sus profesores, señalan reiteradamente que dichos conocimientos no son suficientes para calificar a un académico como «buen docente». Para eso es necesario que también sea capaz de relacionar los contenidos con el mundo laboral, con la coyuntura política o social, con situaciones prácticas generales y que su discurso no sea una mera repetición de los textos preexistentes, sino que se reconozca en él un aporte personal producto de su investigación o experiencia: 
[Es necesario] también una actitud crítica, que no siempre se da en los profesores de la carrera. Es que a mí me ha tocado enfrentarme a muchos profesores que no asumen una actitud crítica y no tienen un buen fundamento para lo que plantean y sus clases son una pura repetición de cosas dichas, en el fondo. O sea, a mí me gusta mucho que el profesor tenga una propia perspectiva sobre los temas que está tratando, que se muestre un compromiso con su trabajo (estudiante Facultad de Filosofía y Humanidades, tercer año).

En consecuencia, en esta área las demandas de los estudiantes parecen perfectamente compatibles con el MFC: se espera un «experto» capaz de significar y acentuar los conocimientos, más que un mero sabio conocedor de innumerables contenidos.

\subsection{Didáctica (clases expositivas vs. múltiples técnicas pedagógicas)}

En el campo de la didáctica, los estudiantes participantes parecen tener una mirada bastante tradicional. Si bien hay algunas referencias a la utilidad de disponer de buena bibliografía, recibir guías de ejercicios, que las presentaciones se apoyen en herramientas audiovisuales y realizar actividades de laboratorio, en general no parece haber una demanda específica para que el docente emplee múltiples técnicas pedagógicas.

Incluso más, en relación con la utilización de herramientas tecnológicas de apoyo a la docencia, si bien no hay un acuerdo discursivo total, predomina la noción de que incluso podrían obstaculizar el aprendizaje, pues los profesores no siempre hacen un buen uso de ellas.

Estoy totalmente en contra de mis profesores que usan el Power Point porque creo que es una forma de descansar sobre el Power Point, o sea, un profesor puede llegar no preparado, pero con un power en un pendrive y puede hacer una clase, no sé cómo, pero después yo veo mis apuntes y veo que son un disparate de ideas (estudiante Facultad de Filosofía y Humanidades, tercer año). 
Así, en el campo de la didáctica, la demanda estudiantil parece centrarse, casi exclusivamente, en clases expositivas tradicionales pero de buena calidad, en las cuales no solo se exponga la información indispensable que no es posible obtener de otras fuentes, sino que principalmente constituyan espacios en los que el académico aplique los contenidos a situaciones concretas, introduzca su particular mirada respecto de los temas, responda las consultas de los estudiantes y ordene y simplifique materias y debates de difícil comprensión.

A mí los profesores que me han gustado son aquellos que para empezar son buenos comunicadores (...) que llaman la atención de los estudiantes, que presentan didácticamente la materia, que la explican, que cambian la entonación, tienen estrategias para que la clase sea de manera didáctica y que el alumno capte la materia (...). Además, muchas veces la materia era muy complicada, una lista enorme de nombres y de relaciones de muchas sustancias químicas, pero que un profesor sepa resumirlo, esquematizarlo y sepa aterrizar la materia es muy bueno para que los estudiantes logren captar de qué se está hablando (estudiante Facultad de Medicina, segundo año).

Estas clases son muchas veces calificadas por los estudiantes como «entretenidas», pero sería un error creer que con esa denominación ellos están haciendo referencia solo a una noción ingenua de diversión, sino que por el contrario, usualmente denominan así la experiencia de haber sido espectadores de una presentación informativa, dinámica, ordenada y provocadora, dictada por un buen comunicador.

Yo creo que un buen profesor siempre es divertido, siempre es entretenido porque un buen profesor supuestamente hace una buena exposición, te da ciertas perspectivas y despierta curiosidades, despierta preguntas (estudiante Facultad de Artes, cuarto año).

En suma, a diferencia de lo propuesto por el MFC, los estudiantes parecen articular una demanda más centrada en mejorar la clase expositiva según sus recuerdos de buenos expositores, que en reemplazarla por otros dispositivos didácticos. 


\subsection{Actitud del docente (seriedad vs. pasión)}

En este tema, los estudiantes demandan un profesor que no solo esté altamente comprometido con su disciplina y las materias que investiga y enseña, sino que además, sea capaz de proyectar y demostrar ese interés a través de su conducta y compromiso docente.

En otras palabras, el profesor ideal debe ser un apasionado por las materias que enseña, capaz de transmitir su entusiasmo a sus estudiantes.

La característica más importante es la transmisión de la pasión frente al conocimiento, frente a lo que te están entregando y que él tenga una actitud provocadora ante los alumnos, o sea, que no solamente sea una transmisión del conocimiento, sino que sus clases, sus palabras, tengan el poder de provocar efectos en mí (...), que él me entregue herramientas también para poder yo desarrollar otra visión (estudiante Facultad de Filosofía y Humanidades, tercer año).

Es importante notar que no es suficiente con que el docente tenga pasión por el tema que trabaja. Para ser un buen profesor también es necesario que este muestre similar interés por la transmisión del conocimiento a sus educandos.

Que tenga una entrega genuina, una vocación genuina, no interesada, que tenga lo menos posible de ego, yo creo, y eso también tiene que ver con la prepotencia de no imponer sus ideas (...) y que el alumno sea escuchado, eso yo creo que es fundamental, que haya motivación y disposición para eso y para eso tiene que haber una vocación (estudiante Facultad de Ciencias, primer año).

En síntesis, en este campo parece haber un completo acuerdo entre los estudiantes y el MFC: se demanda un profesor que proyecte su pasión a los estudiantes. 


\subsection{Interacción con los estudiantes (respeto lejano vs. respeto dialogante)}

En su interacción con los profesores, los estudiantes demandan fundamentalmente una relación dialogante, en la que ellos puedan sentir la confianza necesaria para plantear sus propias opiniones y percepciones respecto de los contenidos tratados y recibir retroalimentación constructiva. Los estudiantes piden tener voz, ser escuchados y ser corregidos, en caso de estar equivocados.

Dentro de las clases los profesores que están dispuestos a contestar tus preguntas y problematizar junto a ti sobre los temas que se están tratando en clase... de nuevo vuelve un poco al tema anterior, del feedback y de la motivación con que uno se presenta en la clase, porque si uno está presente con una persona que uno sabe que te va a escuchar, te va a prestar atención, es súper distinto (estudiante Facultad de Comunicación e Imagen, segundo año).

Ahora bien, para que ese tipo de relación ocurra, tiene que haber un ambiente de respeto mutuo entre el docente y el grupo curso, pues de lo contrario el estudiante se inhibe de participar. Según el discurso de los participantes, el temor a la vergüenza que implica el escarnio público por parte de algún profesor inadecuado es muy alto entre los estudiantes.

Cuando un profesor se burla o es irónico, si yo escucho que a mi compañero, él habló y lo trataron, lo barrieron por el suelo, yo no voy a querer decir algo aunque yo esté seguro que estoy bien, porque yo puedo incluso decir 'oye, yo sé eso que el profesor no sabe', pero no me arriesgo a decírselo porque si él encuentra la manera de ironizarme a mí es terrible" (estudiante Facultad de Filosofía y Humanidades, cuarto año).

El respeto por los estudiantes no solo se refleja en el trato directo, sino también en el nivel de cumplimiento de los compromisos por parte del docente. De esta forma, los comportamientos evaluados como irresponsables son interpretados como una falta de respeto. 
Una primera cosa tiene que ver con cumplir ciertos requisitos mínimos que yo creo que se espera de cualquier persona que trabaja con otras personas o que forma a otras personas, como es el tema de la puntualidad, que sea responsable con ciertas fechas (...) que te traten de manera relativamente respetuosa, con grados de coloquialidad que pueden corresponder de acuerdo a la confianza que tú tienes (estudiante Facultad de Filosofía y Humanidades, tercer año).

Adicionalmente, los estudiantes también exigen que, en un ambiente de respeto y diálogo, el docente sea capaz de mantenerse en su lugar de conductor del proceso educativo, sin comportarse nunca como un par de los estudiantes.

A mí también me ha pasado que hay profes que me cansan, que son demasiado cercanos, y que cuando dejan de hacer su clase pasan a ser un par, eso también encuentro que es un poquito, no sé (estudiante Facultad de Odontología, cuarto año).

En síntesis, los estudiantes parecieran estar demandando casi lo mismo que lo enfatizado por el MFC: la presencia de un docente fuertemente dialogante, pero que igualmente mantenga el control del proceso educativo.

4.6. Evaluación de los aprendizajes (evaluaciones finales válidas vs. evaluaciones formativas y de proceso)

Siendo la evaluación de los aprendizajes uno de los temas más importantes para los estudiantes, se debe señalar que prácticamente no aparecen demandas por ampliar la gama de instrumentos de medición o para que se evalúe el nivel de desarrollo de las competencias a lograr. A lo sumo, aparecen algunas referencias para incorporar evaluaciones más motivadoras que el clásico examen escrito.

Las evaluaciones que también de cierta manera motiven a los estudiantes, por ejemplo, si es que uno hace un trabajo investigativo, es súper interesante aplicar la materia en cierto problema social actual o cierto problema comunicacional actual, a mí me gusta mucho 
hacer ese tipo de trabajos porque puedo aplicar lo que veo teóricamente a nuestra realidad (estudiante Facultad de Comunicación e Imagen, primer año).

Las principales demandas de los estudiantes en este campo tienen relación con la necesidad de recibir una retroalimentación que vaya más allá de la mera calificación obtenida, de modo que el proceso de evaluación tenga un carácter más formativo que sumativo.

Se espera, además, que los instrumentos de evaluación midan conocimientos sustantivos y no solamente memorización de contenidos específicos, así como que cuenten con pautas de correcciones objetivas y claras.

Una pauta, eso, de evaluación, porque las evaluaciones muchas veces son arbitrarias y queda poco claro cuál es el objetivo de esta evaluación, tanto para saber qué te están evaluando y por qué te sacaste esa nota (estudiante Facultad de Filosofía y Humanidades, cuarto año).

Lo que sí encuentro yo es que (...) las cosas que nos preguntan después en las pruebas no son atingentes a nuestro desarrollo profesional, o sea, son más de especialistas (...) se enfocan a cuestiones demasiado específicas que a fin de cuentas no te van a servir nunca (estudiante Facultad de Medicina, cuarto año).

Es interesante notar que no se percibe ningún discurso orientado a disminuir la dificultad de las evaluaciones, sino a hacerlas coherentes con lo trabajado en clases.

Encuentro que tampoco la idea de la universidad es regalar las notas, que todos pasen (...), pero naturalmente tiene que estar eso en concordancia con lo que hizo. O sea, no puede ser un profesor que no hizo nada y después haga una prueba muy difícil. O sea, la clase tiene que tener un carácter que se vea reflejado en la prueba, si la clase es difícil, la prueba tiene que ser más difícil (estudiante Facultad de Filosofía y Humanidades, primer año). 
En suma, si bien las demandas del MFC y de los estudiantes parecen muy similares, el MFC parece ir más allá de lo solicitado por los alumnos al profundizar, tanto en la necesidad de utilizar diversos dispositivos de evaluación, como en la medición de competencias.

\subsection{Resultados esperados (dominio de contenidos disciplinarios vs. desarrollo de competencias generales y específicas)}

En esta área, los estudiantes concuerdan con el MFC en términos de que el profesor no debe limitarse a transmitir contenidos disciplinarios específicos, sino que además, debe intentar producir cambios metacognitivos en sus educandos, transmitiendo nuevos esquemas de interpretación que les permitan entender de manera más elaborada los temas tratados.

Una parte es cómo te enseñan a pensar, eso es lo que yo creo que uno debería aprender en clase, más que la materia en concreto es cómo te enseñan a pensar, y la otra parte que uno tiene que aprender son los datos duros o las cosas que hay, la materia que hay (...) porque en el fondo también para ir desarrollando la forma de pensar es esencial la materia que se sabe, lo que tú estás estudiando (estudiante Facultad de Ciencias, segundo año).

No obstante, en el discurso de los estudiantes no se percibe que estos consideren como tarea fundamental del profesor el desarrollar un abanico de competencias profesionales generales y específicas en sus educandos, sino que concentran sus demandas en la habilidad del docente para generar en los estudiantes capacidades autónomas de reflexión, que les permitan construir una mirada personal respecto del conocimiento.

Yo creo que un profesor tiene que motivar a sus alumnos independientemente del enfoque que él tenga y que motive a los alumnos a seguir más allá, no quedarse con lo que él diga. Yo una vez tuve un profesor [que decía] que no le creyéramos, que no le creyéramos las cosas que él nos decía, que todas las cosas que creyéramos fueran 
cuando las demostrara de verdad, en el fondo eso, que nos motiven a nosotros mismos a ir más allá de las cosas y buscar nuestro propio enfoque de cada materia, de las cosas que estemos buscando (estudiante Facultad de Ciencias Físicas y Matemáticas, segundo año).

En conjunto, parece haber una coincidencia parcial entre los estudiantes y el MFC. Estos parecen estar centrados en el desarrollo de habilidades metacognitivas y en el dominio disciplinario -lo que no es poco-, pero sin plantearse como finalidad de su formación, al menos explícitamente, el desarrollo de mayor diversidad de aprendizajes.

\subsection{Foco del proceso educativo (capacidad del docente para enseñar vs. capacidad del estudiante para aprender)}

Los estudiantes señalan que para ellos la labor pedagógica debe estar centrada exclusivamente en los estudiantes y en su aprendizaje, lo que también exige que los académicos le asignen una relevancia prioritaria a sus actividades docentes dentro del total de sus obligaciones académicas.

Hay profesores que no están cuando les toca, hay profesores que están trabajando en otra parte y no están cuando el alumno lo requiere, cuando les corresponde por horario, hay otros que no están motivados, simplemente dejan al estudiante, 'ya, busquen a un paciente, hagan lo que tienen que hacer'. Sin embargo, también está el otro lado, los docentes que son muy preocupados, que están vigentes, que están ahí todo el tiempo, que incentivan a los estudiantes (estudiante Facultad de Medicina, segundo año).

En cualquier caso, se debe recalcar que en el discurso de los estudiantes la responsabilidad del docente va mucho más allá de la mera generación de un espacio de aprendizaje a través de su curso. Los alumnos asignan al profesor una responsabilidad directa en la generación de aprendizajes efectivos en sus educandos, exigiendo que el profesor no solo enseñe, sino que se asegure de que los estudiantes aprenden. 
Cuando hay vocación hay interés del profesor porque los alumnos aprendan, en el caso del curso de XXX, nadie entendió nada y el profe dijo 'a ver, ¿cómo lo explico?, brubrubrubru, y al final fue lo mismo o peor tal vez, y luego dijo 'bueno, ya, no importa, vamos a seguir con el resto'. Lo que te pasó a ti, tú le pediste ayuda como extraoficialmente y te dijo 'bueno, tal vez este es tu tope, este es tu techo y no vas a superarlo (estudiante Facultad de Filosofía y Humanidades, primer año).

En consonancia con el alto nivel de responsabilidad asignado al docente en el proceso de enseñanza-aprendizaje, prácticamente no aparecen en el discurso estudiantil referencias a la responsabilidad de los alumnos en el proceso de aprendizaje. Por lo tanto, desde su perspectiva, aunque el proceso educativo debe centrarse en el estudiante, la responsabilidad de los aprendizajes parece recaer casi exclusivamente en el profesor.

En consecuencia, en este campo parece haber una coincidencia parcial entre los estudiantes y el MFC: pese a que los primeros demandan que el proceso de enseñanza se centre en ellos, no parecen asumir con claridad la autorresponsabilidad que ello implica en el logro del aprendizaje. En sus discursos el que ellos logren aprender casi siempre parece derivar directamente de la capacidad de sus profesores.

4.9. Nivel de exigencias al docente (exigencias pedagógicas simples vs. exigencias pedagógicas complejas)

Como puede desprenderse de los resultados ya presentados, el discurso de los estudiantes respecto de lo que constituye un buen profesor implica un alto nivel de exigencia para los docentes. Así, los estudiantes esperan de los educadores universitarios importantes capacidades cognitivas, actitudinales, afectivas, relacionales y pedagógicas.

Lo más complejo de este set de demandas no es solo su cantidad y diversidad, sino que se requiere que el docente sea capaz de encontrar el «justo medio» entre demandas polares. Por ejemplo, los educadores deben ser capaces de generar confianza y cercanía 
con sus alumnos, pero no demasiada, como para que la relación no se transforme en una totalmente horizontal; deben realizar evaluaciones de alta exigencia para los alumnos, pero no en exceso; deben programar la asignatura para que cada una de las clases tenga una estructura lógica y ordenada, pero tienen que estar dispuestos a flexibilizarlas para adaptarse a las motivaciones de los estudiantes, entre otras.

Ante este panorama, cabe preguntarse si los estudiantes tienen conciencia de la complejidad de sus demandas y de cómo creen ellos que se puede lograr este tipo de docencia. A este respecto, hay muy pocas referencias en su discurso que apunten a la complejidad del trabajo del docente, por lo que se puede responder negativamente a la primera interrogante.

Respecto de la segunda pregunta, los estudiantes plantean dos sugerencias que implican sendas demandas a la institución universitaria: que los profesores adquieran experiencia docente previamente a tener la responsabilidad de conducir un curso y que se les brinde a los académicos espacios de capacitación en pedagogía.

En mi experiencia propia, los profesores que se han interesado en perfeccionarse en sentido pedagógico se nota demasiado, la preocupación que tienen en ese sentido es mucha y lo transmiten súper bien, entonces es fundamental (estudiante Facultad de Artes, tercer año).

En síntesis, en la actualidad los estudiantes no parecen conformarse con tener como profesores a los mejores expertos en sus disciplinas: hoy demandan también y sobre todo contar con excelentes docentes.

\section{Conclusiones}

Lo primero que esta investigación ha constatado son las altas expectativas que tienen los estudiantes universitarios respecto de sus profesores. No cabe ninguna duda de que los estudiantes están demandando un nuevo tipo de docente, poseedor de un amplio abanico de habilidades. 
Para los estudiantes un docente universitario debe ser capaz de:

- Producir un curso bien organizado, pero adaptable al nivel, heterogeneidad y motivaciones de los alumnos.

- Disponer de un alto y actualizado nivel de conocimientos acerca de la materia que enseña, que le permita discriminar los contenidos relevantes y exponer su propia posición en cada debate.

- Desarrollar clases expositivas altamente motivadoras, informativas, claras y ordenadas.

- Transmitir a sus estudiantes su pasión, tanto por el tema del curso, como por la enseñanza.

- Generar un clima de respeto que permita la participación del alumnado, pero sin entregar el control del proceso educativo.

- Producir evaluaciones justas, válidas, motivadoras, de un nivel de exigencia adecuado y ajustadas a la complejidad de las clases.

- Centrar su trabajo académico en la formación de los estudiantes.

- Responsabilizarse por lograr aprendizajes efectivos y sustantivos en los estudiantes.

¿Es generalizable esta evidencia, o es solo aplicable a los estudiantes de la universidad en la que se realizó la investigación? La mayor parte de los estudios empíricos previos apoya la extensión de estos descubrimientos a otras situaciones. Algunos estudios cuantitativos concluyen que el constructo de «calidad docente» tiene una estructura interrelacionada, pero temáticamente diversa, defendiéndose incluso de que se trata de un concepto con nueve dimensiones (Marsh, 1991). La investigación cualitativa por su parte, también confirma esta pluralidad de demandas. Por ejemplo, Gallardo y Reyes (2010) señalan que los estudiantes solicitan un profesor:

- Cercano afectivamente, capaz de adaptar su clase a los estudiantes y crear espacios para sus opiniones y dudas.

- Entusiasta y centrado en la labor docente.

- Con las habilidades necesarias para transmitir conocimientos complejos.

- Con un manejo de grupo adecuado.

- Capaz de seducir al estudiante por las actividades desafiantes que plantea, por su manejo del tema, su capacidad de ejemplificar y su conocimiento del mundo profesional. 
En la misma línea, Casero (2010) describe múltiples e interconectadas demandas estudiantiles relacionadas con la capacidad del docente de exponer, de ordenar los contenidos, de responder consultas, de tener un conocimiento profundo del tema, de adaptar el curso a los estudiantes, de motivar, de relacionar los contenidos con la práctica, de evaluar en forma justa y coherente, de ser responsable, cercano y respetuoso con sus alumnos, entre otras.

La presencia de altas demandas respecto de la docencia universitaria sin duda constituye una buena noticia, pues implica que los estudiantes presionarán para lograr el mejoramiento de la formación que reciben. Sin embargo, esto puede también constituir un problema si el otro actor clave del proceso, los profesores, no tienen conciencia de las habilidades que se esperan de ellos y de la vara con la que se les mide. Lo anterior puede ser aún más complejo al considerar que una de las explicaciones que los estudiantes construyen para entender por qué existen los malos profesores es atribuirlo a la simple falta de voluntad de estos (existe otra justificación, más constructiva, aunque menos frecuente, que es la falta de capacitación en pedagogía).

Entonces, es posible afirmar que los estudiantes universitarios actuales tienen altas expectativas del desempeño docente de sus profesores, pero ¿son compatibles estas demandas con las establecidas por la formación por competencias?

Del análisis sistemático de los discursos de los estudiantes se desprende que nuestra hipótesis de trabajo, que sostenía la plena coherencia entre el MFC y las expectativas de los alumnos, ha sido apoyada solo en parte por los datos empíricos. De esta forma, si bien hay áreas en las que existe pleno acuerdo entre ambas posiciones, hay otras en las que el acuerdo es solo parcial.

Ejemplos del primer caso son: el énfasis en la necesidad de que el profesor sea respetuoso y cercano a los estudiantes, capaz de exteriorizar su compromiso y pasión por su disciplina y la docencia; la exigencia que se plantea al docente para no ser solo un experto en los contenidos sino que además sepa darles un sentido significativo 
para sus alumnos; o la demanda de altas capacidades pedagógicas a los docentes.

En el segundo caso, es decir, áreas en la que el acuerdo es solo parcial, encontramos: a) en planificación, si bien los estudiantes esperan del profesor la capacidad de adaptarse al contexto y a los estudiantes, prefieren un curso organizado a priori, con una estructura que facilite la comprensión de los temas; b) en evaluación, los estudiantes demandan evaluaciones válidas y formativas, pero no se plantean la necesidad de utilizar distintos formatos de medición; c) en resultados esperados, los estudiantes esperan que sus profesores les transmitan nuevos marcos metacognitivos, pero no que formen competencias profesionales; y d) en responsabilidad del proceso de enseñanza-aprendizaje, los alumnos tienden a centrar la responsabilidad de sus aprendizajes en sus docentes y no en ellos mismos.

En el área de la didáctica incluso es posible afirmar que simplemente no hay acuerdo entre las demandas de los estudiantes y las del MFC, ya que los alumnos solo solicitan que los profesores sean buenos comunicadores y no necesariamente que cambien la clase expositiva por otro dispositivo pedagógico.

La revisión de los campos en los que no hay acuerdo completo entre los estudiantes y MFC permite describir las demandas de los primeros como más tradicionales que las propuestas por este modelo teórico, lo que quizá se explique por la experiencia previa de los estudiantes con sistemas educativos más tradicionales, como podría ser la educación secundaria que han recibido. Como evidencia de ello se puede señalar que, específicamente para el caso chileno, un estudio reciente (Cofré, Camacho, Galaz, Jiménez, Santibáñez y Vergara, 2010) ha constatado que tanto la formación de profesores de ciencias como la docencia que realizan dichos educadores, se puede considerar claramente tradicional, lo que podría contribuir a generar en los estudiantes expectativas conservadoras respecto del comportamiento de sus profesores universitarios.

Puede hipotetizarse que mucha de la discrepancia entre las propuestas del MFC y el discurso de los estudiantes disminuiría si estos recibieran más y mejor información acerca de esta orientación 
curricular y las potencialidades que tiene para su formación y futura empleabilidad. Pero mientras ello no ocurra, es posible que parte de los beneficios de una docencia centrada en el estudiante se pierdan (González, 2010) y los intentos por introducir cambios en la docencia universitaria en línea con lo propuesto por el MFC, enfrenten algunas resistencias por parte del colectivo estudiantil lo que además puede tender a desincentivar a aquellos profesores que más se comprometan con la transformación de su docencia (haciéndola más participativa o empleando mayor proporción de métodos didácticos no expositivos, por ejemplo), al ver que sus esfuerzos no son totalmente comprendidos por sus estudiantes.

Lo anterior implica que si se desea continuar con la implementación del MFC en el ámbito universitario, se deben hacer esfuerzos importantes y sostenidos en el tiempo, para difundir y legitimar este modelo y sus beneficios en la comunidad educativa. Solo si esta legitimación se logra se podrá aspirar a mayores convergencias entre las demandas de los distintos actores del sistema y el modelo por implementar.

De este estudio también se desprende la necesidad de socializar y legitimar ante los profesores las demandas estudiantiles respecto de la docencia, pues no hay evidencia que permita suponer que estos son conscientes de cómo están siendo evaluados por sus alumnos. Es probable que este desconocimiento explique muchos desencuentros entre ambos estamentos.

Finalmente, es necesario destacar que uno de los principales aportes de esta investigación constituye a la vez una de sus más importantes limitaciones, ya que al ser el primer estudio que realiza una comparación sistemática entre las demandas estudiantiles y las del MFC, no es posible determinar si sus resultados pueden ser extrapolables a otros contextos o son dependientes de la especificidad de la situación estudiada: alumnos de una misma universidad, con alto prestigio en el contexto latinoamericano y que, por lo tanto, tienen altas expectativas acerca de sus aprendizajes. Es aconsejable replicar el estudio en otras condiciones para determinar si es posible mantener sus conclusiones. 


\section{Referencias}

Atlas.ti Scientific Software, (2004) Atlas.ti: the knowledge workbench (5.0). Berlin, Scientific Software.

Bedek, A. y A. Urbanc, (2009) «Quality of higher education: in search for ideal professor from educational and students perspective» ponencia presentada en la First International Student Conference, Zagreb, Croacia, 12 de junio de 2009. Disponible en: https://sites.google.com/site/ isc2009croatia/download-zone-1

Bozu, Z. y P.J. Canto, (2009) «El profesorado universitario en la sociedad del conocimiento: competencias profesionales docentes». Revista de Formación e Innovación Educativa Universitaria, 2(2), pp. 87-97.

Casero, A., (2010) «¿Cómo es el buen profesor universitario según el alumnado?» Revista Española de Pedagogía, 68(246), pp. 223-242.

Chonko, L.; Janner, J. y R. Davies, (2002) «What are thinking? Students expectations and self-assessments». Journal of Educational for Business, 77(5), pp. 271-281.

Cofré, H.; Camacho, J.; Galaz, A.; Jiménez, J.; Santibáñez, D. y C. Vergara, (2010) «La educación científica en Chile: debilidades de la enseñanza y futuros desafíos de la educación de profesores de ciencia». Estudios Pedagógicos, 36(2), pp. 279-293.

Dávila, A., (1993) «Las perspectivas metodológicas cualitativa y cuantitativa en las ciencias sociales: debate teórico e implicaciones fraseológicas. En J.M. Delgado y J. Gutiérrez (eds.) Métodos y técnicas cualitativas de investigación en ciencias sociales» (pp. 69-83). Madrid, Síntesis.

De Miguel, M., (2005) «Cambio de paradigma metodológico en la educación superior: exigencias que conlleva». Cuadernos de Integración Europea, 2, pp. 16-27.

De Miguel, M., (2006) Modalidades de enseñanza centradas en el desarrollo de competencias: orientaciones para promover el cambio metodológico en el Espacio Europeo de Educación Superior (EEES), Oviedo, Universidad de Oviedo.

Díaz-Barriga, A., (2006) «El enfoque de competencias en la educación, ¿una alternativa o disfraz de cambio?». Perfiles Educativos, 28(111), pp. 7-36.

Fernández-Salinero, C., (2006) «Las competencias en el marco de la convergencia europea: un nuevo concepto para el diseño de programas educativos». Encounters on Education, 7, pp. 131-153.

Francis, S., (2006) «Hacia una caracterización del docente universitario "excelente": una revisión a los aportes de la investigación sobre el 
desempeño del docente universitario». Revista Educación, 30(1), pp. 31-39.

Gallardo, G. y P. Reyes, (2010) «Relación profesor-alumno: arista fundamental para el aprendizaje». Revista Calidad de la Educación, 32, pp. 78-108.

Gargallo, B.; Sánchez, F.; Ros, C. y A. Ferreras, (2010) «Estilos docentes de los profesores universitarios. La percepción de los alumnos de los buenos profesores». Revista Iberoamericana de Educación, 51(4), pp. 1-16.

Giné, N., (2008) «Cómo mejorar la docencia universitaria: el punto de vista del estudiantado». Revista Complutense de Educación, 20(1), pp. 117-134.

Gómez, M.A. y M.V. Alzate, (2010) «La alegre entrada y el irresistible ascenso de las competencias en la universidad». Educación y Educadores, 13(3), pp. 453-474.

González, C., (2010) «El aprendizaje y el conocimiento académico sobre la enseñanza como claves para mejorar la docencia universitaria». Revista Calidad en la Educación, 33, pp. 123-146.

Greimel-Fuhrmann, B. y A. Geyer, (2003) «Students' evaluation of teachers and instructional quality-analysis of relevant factors based on empirical research». Assessment and Evaluation in Higher Education, 28(3), pp. 229-238.

Jahangiri, L. y T. Mucciolo, (2008) «Characteristics of effective classroom teachers as identified by students and professionals: a qualitative study». Journal of Dental Education, 72(4), pp. 707-718.

Marsh, H., (1991) «Multidimensional students evaluations of teaching effectiveness: a test of alternative higher-order structures». Journal of Educational Psychology, 83(2), pp. 285-296.

Morgan, D.L., (1990) Focus groups as qualitative research. Newbury Park, Sage.

Okpala, C. y R. Ellis, (2005) «The perceptions of college students on teacher quality: a focus on teacher qualifications». Education, 126(2), pp. 374-383.

Pozo, C.; Rebolloso, E. y B. Fernández, (2000) «The "ideal teacher". Implications for student evaluation of teacher effectiveness». Assessment and Evaluation in Higher Education, 25(3), pp. 253-263.

Tardif, J., (2003) «Développer un programme par compétences: de l'intention à la mise en oeuvre». Pedagogie Collégiale, 16(3), pp. 36-44.

Taylor, S. y R. Bogdan, (1992) Introducción a los métodos cualitativos de investigación. Barcelona, Paidós. 
Tejada, J., (2002) «El docente universitario ante los nuevos escenarios: implicaciones para la innovación docente». Acción Pedagógica, 11(2), pp. 30-42.

Tejada, J., (2009) «Competencias docentes». Profesorado, Revista de Currículum y Formación del Profesorado, 13(2), pp. 1-15.

Valcarcel, M., (2005) La preparación del profesorado universitario para la convergencia europea en educación superior. Informe de investigación no publicado, proyecto ea2003-0040.

Van Vucht Tijssen, L. y E. de Weert, (2005) «From erudition to academic competence». Revista Española de Pedagogía, 63(230), pp. 123-146.

Villa, A. y O. Villa, (2007) «El aprendizaje basado en competencias y el desarrollo de la dimensión social en las universidades». Educar, 40, pp. 15-48.

Zabalza, M.A., (2003) Competencias docentes del profesorado universitario. Calidad y desarrollo profesional. Madrid, Narcea.

Recibido: 18/03/2013

Aceptado: 31/05/2013 